\title{
Outage Performance of Cognitive Relay Networks with Best Relay Selection in Nakagami-m Channels
}

\author{
Zongsheng Zhang, Qihui Wu, Jinlong Wang, Xueqiang Zheng, Xinhong Shao, Cheng Tan \\ Affiliation: College of Communications Engineering, PLA University of Science and Technology, \\ No. 2, YuDao Street, Nanjing China \\ Email: zhangzongsheng1984@163.com
}

Received May, 2013

\begin{abstract}
This paper investigates the outage performance of a cognitive relay network considering best relay selection in Nakagami-m fading environment. The secondary user is allowed to use the spectrum when it meets the interference constraints predefined by primary user. Due to deep fading, cognitive source is unable to communicate directly with cognitive destination. As such, multiple relays are ready to deliver the signal from the cognitive source to cognitive destination. We select a single best relay and the selected relay uses decode-and-forward protocol. Specifically, we derive the exact outage probability expression, which provides an efficient means to evaluate the effects of several parameters. Finally, numerical simulation results are presented, which validate the correctness of the analytical analysis.
\end{abstract}

Keywords: Cognitive Realy Networks (CRNs); Nakagami-m Fading; Outage Probability

\section{Introduction}

In the past decades, the demand for wireless spectrum use has been growing dramatically with the rapidly development of the mobile telecommunication industry. Conventional spectrum management policies use static spectrum assignment to prevent interference. Recently, this policy has led to the well-known artificial spectrum scarcity. Lots of surveys have told us that the licensed spectrum are critically under-utilized, often as low as $2 \%-15 \%$ [1]. This motivates the concept of spectrum reuse that allows secondary users (SUs) to re-use the spectrum. The key technology behind spectrum re-use is cognitive radio (CR) [2-5]. The CR technology is defined as a technology that can guide the communication system to adjust its power, frequency, modulation, coding, and other parameters to efficiently utilize the licensed spectrum.

Cooperative technology, emerging as a new spatial diversity technique, can effectively combat fading and improve the throughput. However, the advantages of such system achieve at the expense of a reduction in spectral efficiency. As such, relay selection has been investigated [6,7] to overcome this shortcoming. Recently, cooperation also has great potential to be used in cognitive radio networks, known as cognitive relay networks (CRNs) [8-14]. In [11], the exact outage probability of an underlay CRNs using DF relaying with multiple PUs in Rayleigh fading channels has been derived. Most recently, the exact outage probability of an underlay cognitive relay networks over Nakagami-m fading was derived in [12]. In [13], the outage probability of cognitive amplify-and-forward (AF) relay networks in independent non-identically distributed (i. n. i. d) Nakagami-m fading was investigated. The outage probability of dual-hop CRNs considering the direct link and interference from primary user has been derived in [14]. In [15], the outage performance of CRNs considering both direct link and relay selection was investigated in Rayleigh fading environment. The outage analysis of amplify-and-forward with partial relay selection under spectrum-sharing constraints was analyzed in [16].

While those previous work have improved our understanding on the outage performance of CRNs. However, the previous work [11-14] ignored the relay selection, and $[15,16]$ considered the Rayleigh fading environment. To the best of our knowledge, the outage performance of CRNs considering relay selection in Nakagami-m fading environment is almost unexplored from the analytical point view. As such, the main focus of this paper is to fill this important gap.

The remainder of this paper is organized as follows. Section II presents a brief description of system model. In Section III, the exact outage probability expression of the considered system is derived. Some numerical results are presented to validate the correctness of theoretical analysis in Section IV. Finally, some concluding remarks are provided in Section V. 


\section{System Model}

We consider a spectrum-sharing system with one pair of primary user ${ }^{1}$, a cognitive source (S), M cognitive relays ${ }^{2}$, and a cognitive destination (D), as depicted in Figure 1. The communication in cognitive system takes place in two phases. In the first phase, the cognitive source broadcasts the signal to cognitive relays. In the second phase, the best relay decodes the signal and forwards the decoded signal to the cognitive destination. Specifically, all the channel gains between any two nodes are Nakagami-m fading.

Specifically, for the transmission of cognitive system, the cognitive source and cognitive relay should limit their transmit powers so that the interference on the primary network will not exceed a threshold $Q$, which is the peak interference that the primary network can tolerate. As such, the transmit power of the cognitive source and cognitive relay can be expressed as

$$
P_{S}=\frac{Q}{\left|h_{3}\right|^{2}}
$$

and

$$
P_{R_{i}}=\frac{Q}{\left|h_{4 i}\right|^{2}}
$$

where $\left|h_{3}\right|^{2}$ and $\left|h_{4 i}\right|^{2}$ denote the channel gains of $S \rightarrow P U$ and $R_{i} \rightarrow P U$, respectively. Specifically, the channel gains $\left|h_{1 i}\right|^{2},\left|h_{2 i}\right|^{2},\left|h_{3}\right|^{2}$ and $\left|h_{4 i}\right|^{2}$ follow a Nakagami-m distribution with fading parameters $m_{i}$ and $\Omega_{i}, \quad i=1,2,3,4$. Therefore, the probability density function (PDF) and cumulative distributed function (CDF) of a gamma random variable with parameters $m$ and $\Omega$ can be denoted as

$$
f_{|h|^{2}}(t)=\frac{\beta^{m}}{\Gamma(m)} t^{m-1} e^{-\beta m}
$$

and

$$
F_{|h|^{2}}(t)=\frac{\gamma(m, \beta t)}{\Gamma(m)}
$$

where $\beta=m / \Omega, \gamma(m, t)$ represents the incomplete gamma function [17]. Consequently, the received signal-to-interference ratio (SIR) at the ith relay and cognitive destination can be expressed as

$$
\gamma_{S R_{i}}=\frac{Q}{\left|h_{3}\right|^{2}} \frac{\left|h_{1 i}\right|^{2}}{N_{0}}
$$

\footnotetext{
${ }^{1}$ In this paper, we only consider a single primary user, for simplicity of analysis. The obtained results can be extended to multiple primary user.

${ }^{2}$ In this paper, multiple relays are assumed closely located to be a cluster. Therefore, we assume that the distance between any relay are small compared to the distance between cognitive source and relay or between relay and cognitive destination. As such, for simplicity of analysis, the pathloss is same for any relays, and is not taken into consideration in this paper.
}

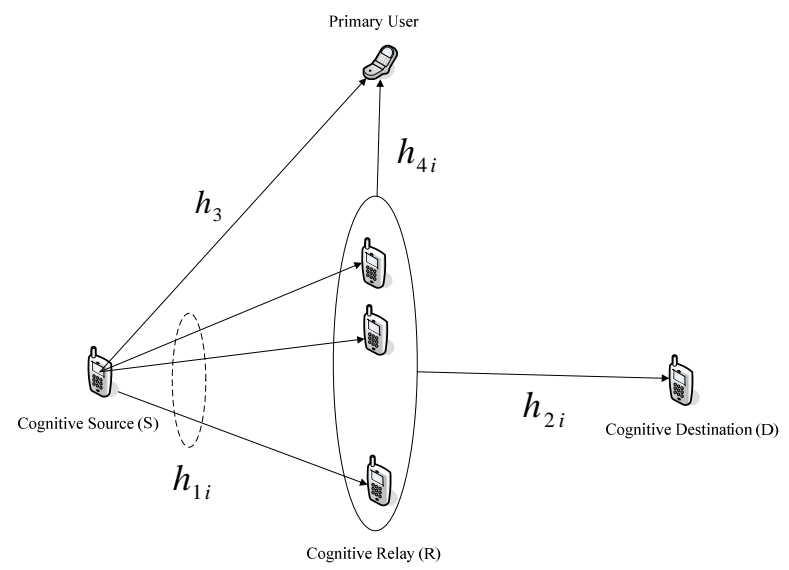

Figure 1. System model.

and

$$
\gamma_{R_{i} D}=\frac{Q}{\left|h_{4 i}\right|^{2}} \frac{\left|h_{2 i}\right|^{2}}{N_{0}}
$$

where $N_{0}$ denotes the variance of additive white Gaussian noise (AWGN). For simplicity of analysis, we set $N_{0}=1$. Because we select the best decode-and-forward protocol in this paper. As such, the end-to-end SIR can be denoted as

$$
\gamma^{S-D F}=\max _{i=1, \ldots, M}\left\{\min \left(\gamma_{S R_{i}}, \gamma_{R_{i} D}\right)\right\}
$$

\section{Outage Probability}

In this section, we will derive the outage probability of the considered system. The outage probability, i.e., the end-to-end SIR falls below the threshold $\gamma_{t h}$, is expressed as

$$
P_{\text {out }}=\operatorname{Pr}\left\{\gamma^{B-D F} \leq \gamma\right\}=F_{\gamma^{B-D F}}(\gamma)
$$

Accordingly, the main focus is to derive the CDF of $\gamma^{B-D F}$. Based on (5) and (6), we conclude that the $\gamma_{S R_{i}}$ and $\gamma_{R_{i} D}$ are not independent due to common variable $\left|h_{3}\right|^{2}$. For simplicity of representation, we set $X=\left|h_{3}\right|^{2}$,

$$
A=\left|h_{1 i}\right|^{2}, \quad B=\left|h_{2 i}\right|^{2}, C=\left|h_{4 i}\right|^{2}
$$

and

$$
U=\min \left(\gamma_{S R_{i}}, \gamma_{R_{i} D}\right) \text {. }
$$

Therefore, the CDF of $U$ conditioned on $X$ can be expressed as

$$
\begin{aligned}
F(U \mid X) & =\operatorname{Pr}\left\{\min \left(\gamma_{S R_{i}}, \gamma_{R_{i} D}\right) \leq \gamma \mid X\right\} \\
& =1-\operatorname{Pr}\left\{\min \left(\gamma_{S R_{i}}, \gamma_{R_{i} D}\right) \geq \gamma \mid X\right\} \\
& =1-\left(1-F_{\gamma_{S R_{i}}}(\gamma \mid X)\right)\left(1-F_{\gamma_{R_{i} D}}(\gamma \mid X)\right)
\end{aligned}
$$

As such, the main focus is to derive the $F_{\gamma_{S R_{i}}}(\gamma \mid X)$ and $F_{\gamma_{R_{i} D}}(\gamma \mid X)$. In this way, the $F_{\gamma_{S_{i}}}(\gamma \mid X)$ and $F_{\gamma_{R_{i} \mathrm{D}}}(\gamma \mid X)$ can be expressed as 


$$
\begin{aligned}
F_{\gamma_{S R_{i}}}(\gamma \mid X) & =\operatorname{Pr}\left\{\frac{Q\left|h_{1 i}\right|^{2}}{N_{0} X} \leq \gamma \mid X\right\} \\
& =\operatorname{Pr}\left\{A \leq \frac{\gamma X}{Q} \mid X\right\} \\
& =\frac{\gamma\left(m_{1}, \frac{\beta_{1} \gamma X}{Q}\right)}{\Gamma\left(m_{1}\right)}
\end{aligned}
$$

and

$$
\begin{aligned}
F_{\gamma_{R_{i}}}(\gamma \mid X) & =\operatorname{Pr}\left\{\frac{Q\left|h_{2 i}\right|^{2}}{\left|h_{4 i}\right|^{2}} \leq \gamma \mid X\right\} \\
& =\operatorname{Pr}\left\{B \leq \frac{\gamma C}{Q} \mid X\right\} \\
& =\int_{0}^{\infty} f_{C}(c) \int_{0}^{\frac{\gamma c}{Q}} f_{B}(b) d b d c \\
& =\int_{0}^{\infty} f_{C}(c) F_{B}\left(\frac{\gamma C}{Q}\right) d c \\
& =\int_{0}^{\infty} \frac{\beta_{4}{ }^{m_{4}}}{\Gamma\left(m_{4}\right)} c^{m_{4}-1} e^{-\beta_{4} c} \frac{\gamma\left(m_{2}, \frac{\beta_{2} \gamma c}{Q}\right)}{\Gamma\left(m_{2}\right)} d c \\
& =1-I_{1}
\end{aligned}
$$

where

$$
I_{1}=\frac{\beta_{4}^{m_{4}}}{\Gamma\left(m_{4}\right)} \sum_{k_{1}=0}^{m_{2}-1} \frac{\left(\beta_{2} \gamma\right)^{k_{1}}}{Q^{k_{1}} k_{1} !} \frac{\left(m_{4}+k_{1}-1\right) !}{\left(\beta_{4}+\frac{\beta_{2} \gamma}{Q}\right)^{-\left(m_{4}+k_{1}\right)}}
$$

As such, the $F_{U}(\gamma \mid X)$ can be denoted as

$$
\begin{aligned}
F_{U}(\gamma \mid X) & =1-\left(1-F_{\gamma_{S R_{i}}}(\gamma \mid X)\right)\left(1-F_{\gamma_{R_{i} D}}(\gamma \mid X)\right) \\
= & 1-\left(1-F_{\gamma_{S R_{i}}}(\gamma \mid X)\right) I_{1} \\
& =1-\left(1-\frac{\gamma\left(m_{1}, \frac{\beta_{1} \gamma X}{Q}\right)}{\Gamma\left(m_{1}\right)}\right) I_{1}
\end{aligned}
$$

Due to independent distribution and random variable $\gamma^{B-D F}$ is the maximum of $M$ random variables. Therefore, the CDF of $\gamma^{B-D F}$ conditioned on $X$ can be expressed as

$$
F_{\gamma^{B-D F}}(\gamma \mid X)=\left(1-\left(1-\frac{\gamma\left(m_{1}, \frac{\beta_{1} \gamma X}{Q}\right)}{\Gamma\left(m_{1}\right)}\right) I_{1}\right)^{M}
$$

Based on (14), the unconditional cumulative distributed function of $\gamma^{B-D F}$ marginalized out with respect to $X$ can be calculated as

$$
F_{\gamma^{S-D F}}(\gamma)=\int_{0}^{\infty} F_{\gamma^{B-D F}}(\gamma \mid X) f_{X}(x) d x
$$

Substituting (3) and (14) into (15), the $F_{\gamma^{B-D F}}(\gamma)$ can be calculated as

$$
\begin{aligned}
& F_{\gamma S-D F}(\gamma) \\
= & \int_{0}^{\infty} F_{\gamma^{B-D F}}(\gamma \mid X) f_{X}(x) d x \\
= & \int_{0}^{\infty}\left(1-\left(1-\frac{\gamma\left(m_{1}, \frac{\beta_{1} \gamma x}{Q}\right)}{\Gamma\left(m_{1}\right)}\right) I_{1}\right)^{M} \frac{\beta_{3}^{m_{3}}}{\Gamma\left(m_{3}\right)} x^{m_{3}-1} e^{-\beta_{3} x} d x \\
= & \int_{0}^{\infty} \sum_{m=0}^{M} C_{M}^{m}\left(-I_{1}\right)^{m}\left(1-\frac{\gamma\left(m_{1}, \frac{\beta_{1} \gamma x}{Q}\right)}{\Gamma\left(m_{1}\right)}\right)^{m} \frac{\beta_{3}^{m_{3}}}{\Gamma\left(m_{3}\right)} x^{m_{3}-1} e^{-\beta_{3} x} d x
\end{aligned}
$$$$
\text { The }\left(1-\frac{\gamma\left(m_{1}, \frac{\beta_{1} \gamma x}{Q}\right)}{\Gamma\left(m_{1}\right)}\right)^{m} \text { can be expanded as follows }
$$$$
\left(1-\frac{\gamma\left(m_{1}, \frac{\beta_{1} \gamma x}{Q}\right)}{\Gamma\left(m_{1}\right)}\right)^{m}=e^{-\frac{\beta_{1} \gamma m x}{Q}} \sum_{l_{1}=0}^{m_{1}-1} \cdot \sum_{l_{m}}^{m_{1}-1} \frac{\left(\frac{\beta_{1} \gamma x}{Q}\right)^{l_{1}+\ldots+l_{m}}}{l_{1} ! \ldots l_{m} !}
$$

Plugging (17) into (16), the exact outage expression can be expressed as

$$
\begin{aligned}
& F_{\gamma-D F}(\gamma) \\
= & \int_{0}^{\infty} \sum_{m=0}^{M} C_{M}^{m}\left(-I_{1}\right)^{m} e^{\frac{\beta_{\gamma} m x^{2}}{Q}} \sum_{l_{1}=0}^{m_{1}-1} \cdot \sum_{l_{m}}^{m_{1}-1} \frac{\left(\frac{\beta_{1} \gamma x}{Q}\right)^{l_{1}+\ldots l_{m}}}{l_{1} ! \ldots l_{m} !} \frac{\beta_{3}^{m_{3}}}{\Gamma\left(m_{3}\right)} x^{m_{3}-1} e^{-\beta_{3} x} d x \\
= & \sum_{m=0}^{M} C_{M}^{m}\left(-I_{1}\right)^{m} \sum_{l_{1}=0}^{m_{1}-1} \cdot \sum_{l_{m}}^{m_{1}-1} \frac{\left(\frac{\beta_{1} \gamma}{Q}\right)^{l_{1}+.+l_{m}}}{l_{1} ! \ldots l_{m} !} \frac{\left(l_{1}+\ldots+l_{m}+m_{3}-1\right) !}{\left(\beta_{3}+\frac{\beta_{1} \gamma m}{Q}\right)^{\left(l_{1}+.+l_{m}+m_{3}\right)}}
\end{aligned}
$$

\section{Numerical Results}

In this section, we confirm the analytical results derived in Section III via comparisons using Monte Carlo simulations. We mainly focus on the impacts of interference links, transmit links and the maximum allowable transmit power of primary user on the outage performance of the considered system. Specifically, all the simulation results are obtained through $10^{8}$ independent trials.

Firstly, we evaluate the impact of the number of relays on the outage performance of the cognitive relay networks. The parameters for simulation are set as follows: $m_{1}=1, m_{2}=1, m_{3}=1$ and $m_{4}=1$. Specifically, we consider three schemes in the simulation: Scheme 1: $M=1$, Scheme 2: $M=3$, Scheme 3: $M=5$. Obviously, Figure 2 shows that the outage performance of the considered system will improve when we increase $Q$. Similarly, the same conclusion can be obtained from the parameters $M$ and the $M$ affects the diversity of the considered system. Moreover, the Monte Carlo simulation results keep tight with the analytical results which validate the analytical analysis.

Secondly, we evaluate the impact of channel gains of 
the secondary system on the outage performance of the cognitive relay networks. As such, the channels gains between the secondary user and the primary user keep fixed. We consider two schemes in the simulation: Scheme 1: $m_{i}=(1,1,1,1)$, Scheme 2: $m_{i}=(2,2,1,1)$, $i=1,2,3,4$. Figure 3 clearly shows that the outage performance will improve greatly when we increase the quality of channel gains of the secondary system. specifically, the channel gains of the secondary system will not affect the diversity of the considered system.

Lastly, we evaluate the impact of the channel gains from the primary user on the outage performance of the cognitive relay networks. As such, the channel gains between secondary system keep fixed. Similarly, we also consider two schemes in the simulation: Scheme 1: $m_{i}=(2,2,2,2)$, Scheme 2: $m_{i}=(2,2,1,1), i=1,2,3,4$. We can directly observe from Figure 4 that the outage probability will increase when the quality of primary links becomes better. Moreover, the channel gains between secondary user and primary user do not affect the diversity of the considered system.

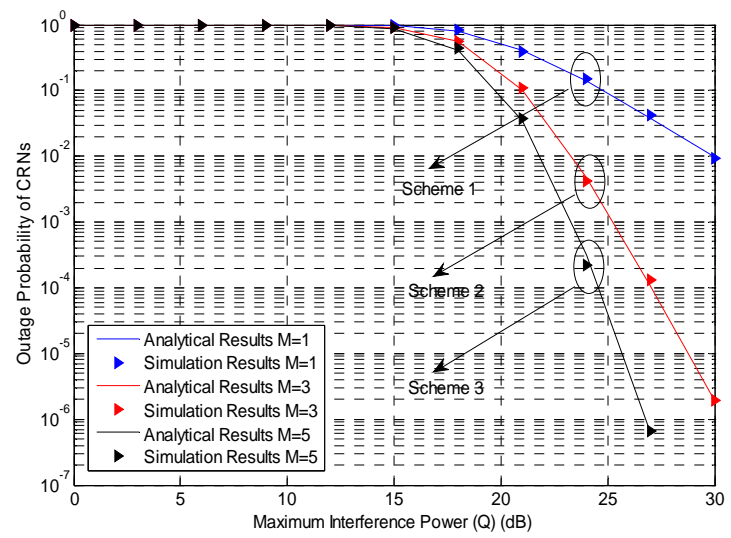

Figure 2. Impacts of number of relays on the outage performance of cognitive relay networks.

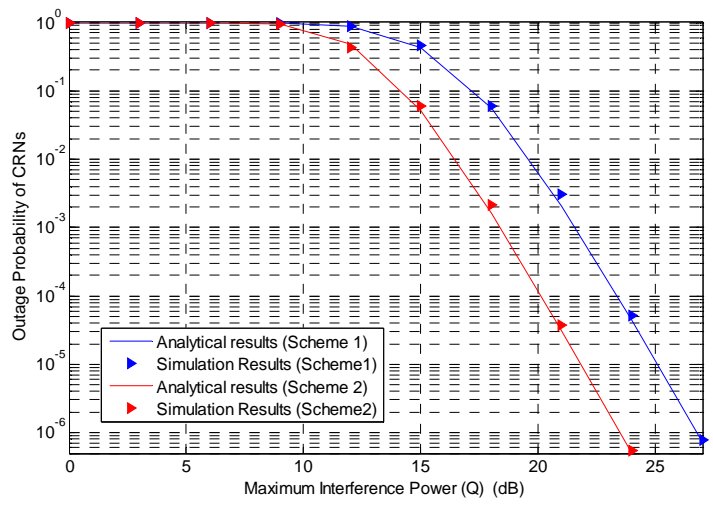

Figure 3. Impacts of channel gains of the secondary system on the outage performance of cognitive relay networks.

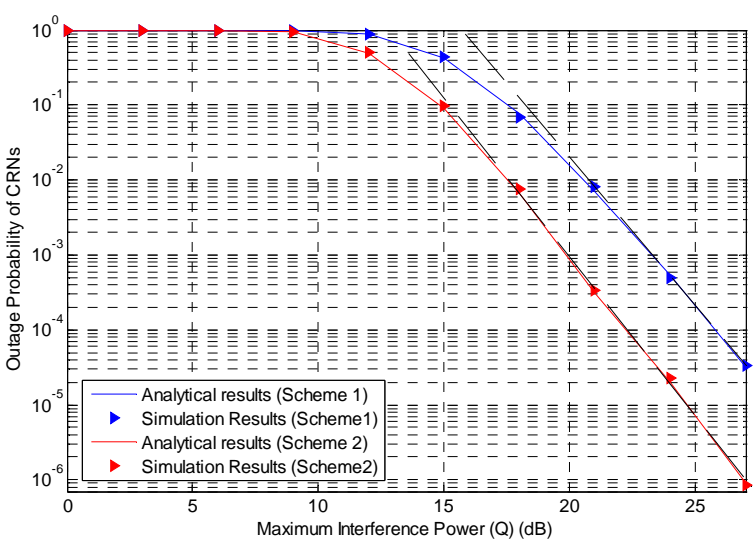

Figure 4. Impacts of channel gains of primary network on the outage performance of cognitive relay networks.

\section{Conclusion}

In this paper, the outage performance of cognitive relay networks with multiple relays was derived in Nakagamim fading environment. We consider a spectrum-sharing system with a primary user as long as the secondary user meets the power interference requirements predefined by the primary user. Specifically, the best decode-and-forward relay selection protocol is adopted in this paper. Moreover, we derived the exact outage probability expression, which provides an efficient means to evaluate the effects of several parameters. Last, numerical results are provided, and the Monte Carlo simulations results match well with the analysis results.

\section{Acknowledgements}

This work was supported by the national basic research program (973) of China under grant no. 2009CB320400, the National Science Foundation of China under grant no. 60932002 and no. 61172062, and in part by the Jiangsu Province Natural Science Foundation of China under grant no. BK2011116.

\section{REFERENCES}

[1] Federal Communication Commission (FCC). ET Docket No 03-322: Notice of Proposed Rule Making and Order in the Matter of Facilitating Opportunities for Flexible, Efficient, and Reliable Spectrum Use Employing Cognitive Radio Technologies. Washington DC (USA), 2003.

[2] Z. Zhang, Q. Wu and J. Wang, "Energy-efficient Power Allocation Strategy in Cognitive Relay Networks,” Radio Engineering, Vol. 21, No. 3, 2012, pp. 809-814.

[3] Y. Xu, et al., "Opportunistic Spectrum Access in Unknown Dynamic Environment: A Game-theoretic Stochastic Learning Solution," IEEE Transactions on Wireless Commun., Vol. 11, No. 4, 2012, pp. 1380-1391, 2012.

[4] J. Mitola, Cognitive Radio: An Integrated Agent Archi- 
tecture for Software Defined Radio. Ph. D. Dissertation. Stockholm (Sweden): Royal Institute of Technology (KTH), 2000.

[5] Y. Zou, et al., "Cooperative Relay Techniques for Cognitive Radio Systems: Spectrum Sensing and Secondary User Transmission,” IEEE Communications Magazine, Vol. 50, No. 4, 2012, pp. 98-103.doi:10.1109/MCOM.2012.6178840

[6] S. ikki and M. H. Ahmed, "Performance Analysis of Adaptive Decode-and-forward Cooperative Diversity Networks with Best Relay Selection,” IEEE Transactions Communications, Vol. 58, No. 1, 2010, pp. 68-72. doi:10.1109/TCOMM.2010.01.080080

[7] A. Bletsas, H. Shin, M. Z. Win and A. Lippman, "A Simple Cooperative Diversity Method Based on Network Path Selection,” IEEE J. Sel. Areas Commun., Vol. 24, No. 3, 2006, pp. 659-672.doi:10.1109/JSAC.2005.862417

[8] Q. Wu, Z. Zhang and J. Wang, “Outage Analysis of Cognitive Relay Networks with Relay Selection under Imperfect CSI Environment,” IEEE Commun. Letters.

[9] J. Wang, Z. Zhang, Q. Wu and Y. Huang, “Outage Analysis of Cognitive Relay Networks with Interference Constraints in Nakagami-m Channels," IEEE Wireless Commun. Letters.

[10] Y. Zou, et al., "Cooperative Relay Techniques for Cognitive Radio Systems: Spectrum Sensing and Secondary User Transmissions," IEEE Communications Magazine, Vol. 50, No. 4, 2012, pp. 98-103. doi:10.1109/MCOM.2012.6178840

[11] T. Q. Duong, et al., "Cognitive Relay Networks with
Multiple Primary Transceivers under Spectrum-Sharing," IEEE Signal Processing Letters, Vol. 19, No. 11, 2012, pp. 741-744. doi:10.1109/LSP.2012.2217327

[12] C. Zhong, et al., "Outage Analysis of Decode-and-forward Cognitive Dual-hop Systems with the Interference Constraint in Nakagami-m Fading Channels,” IEEE Transactions Vehicular Technology, Vol. 60, No. 5, 2012, pp. 2368-2374.

[13] T. Q. Duong, et al., "Cognitive Amplify-and-forward Relay Networks over Nakagami-m Fading," IEEE Transactions Vehicular Technology, Vol. 61, No. 5, 2012, pp. 2368-2374. doi:10.1109/TVT.2012.2192509

[14] T. Q. Duong, et al., "Outage and Diversity of Cognitive Relaying Systems under Spectrum Sharing Environments in Nakagami-m fading,” IEEE Communications Letters, Vol. 16, No. 12, 2012, pp. 2075-2078. doi:10.1109/LCOMM.2012.100812.121859

[15] L. Luo, P. Zhang, G. Zhang and J. Qin, “Outage Performance for Cognitive Relay Networks with Underlay Spectrum Sharing," IEEE Communications Letters, Vol. 15, No. 7, 2011.pp.710-712 doi:10.1109/LCOMM.2011.051011.110426

[16] K. B. Fredj, et al., "Performance of amplify-and-forward systems with partial relay selection under spectrum-haring constraints," IEEE Transactions Wireless Commun., Vol. 11, No. 2, 2012.

[17] I. S. Gradshteyn and I. M. Ryzhik, Table of Integrals, Series, and Products, $5^{\text {th }}$ ed. Orlando, FL: Academic, 1994. 